\title{
Crecimiento guiado con barras magnéticas en pacientes con escoliosis de inicio temprano. Reporte preliminar
}

\author{
César Orellana, Mariano Noel, Ernesto Bersusky, Rodrigo Remondino, Eduardo Galaretto, \\ Carlos Tello, Lucas Piantoni, Ida A. Francheri Wilson \\ Servicio de Patología Espinal, Hospital de Pediatría "Prof. Dr. Juan P. Garrahan", \\ Ciudad Autónoma de Buenos Aires, Argentina
}

\begin{abstract}
RESUMEN
Introducción: El tratamiento de las escoliosis de inicio temprano guiado con barras magnéticas permite realizar distracciones no invasivas y ambulatorias. El objetivo de este estudio fue evaluar nuestra primera serie de casos con escoliosis de inicio temprano tratados con el sistema de barras magnéticas. Materiales y Métodos: Se realizó una revisión de casos tratados con el sistema de barras magnéticas entre 2014 y 2018. Se formaron dos grupos: grupo I (procedimientos primarios con barras magnéticas) y grupo II (conversiones de sistema tradicional a barras magnéticas). Resultados: Se evaluó a 19 pacientes. La edad promedio en el momento de la cirugía era de 7 años y 4 meses, con un seguimiento promedio de 2 años y 7 meses. El grupo I tenía 12 pacientes y el grupo II, 7 pacientes. Los valores angulares promedio preoperatorio y posoperatorio inmediato de la escoliosis fueron $62^{\circ}$ y $42^{\circ}$, respectivamente; los de cifosis, $49^{\circ}$ y $34^{\circ}$, respectivamente. La distancia T1-T12 fue de 160 a $176 \mathrm{~mm}$. La distancia T1-S1 fue de 285 a 317 mm. Hubo una complicación: protrusión del implante e infección, y fue necesario retirar el material (grupo II). Conclusiones: Los resultados preliminares sugieren que es un método seguro y eficaz. Si bien los resultados a corto y mediano plazo son alentadores, persisten algunos desafíos importantes e incógnitas en relación con el comportamiento mecánico del implante en un seguimiento prolongado.
\end{abstract}

Palabras clave: Escoliosis de inicio temprano; barras de crecimiento; barras magnéticas.

Nivel de Evidencia: IV

\section{Guided Growth with Magnetic Rods in Early Onset Scoliosis. Preliminary Report}

\section{ABSTRACT}

Introduction: Early onset scoliosis (EOS) treatment with the magnetically controlled growing rod (MCGR) system allows for the use of non-invasive outpatient distractions. The purpose of this study was to assess our first series of EOS patients treated with MCGRs. Materials and methods: We conducted a review of EOS cases treated with MCGRs between 2014 and 2018 . The study population was divided into two groups: Group I, patients undergoing primary MCGR insertion; Group II, patients undergoing conversion from conventional growth system to MCGR. Results: The study population consisted of 19 patients. The average age at the time of surgery was 7 years and 4 months, with an average post-operative follow-up of 2 years and 7 months. Group I consisted of 12 patients and Group II of 7 patients. The mean preoperative scoliosis angle was $62^{\circ}$ and immediate postoperatively was $42^{\circ}$. The mean preoperative kyphosis angle was $49^{\circ}$ and immediate postoperatively was $34^{\circ}$. The average preoperative $\mathrm{T} 1-\mathrm{T} 12$ length was $160 \mathrm{~mm}$ and immediate postoperatively was $176 \mathrm{~mm}$. The average preoperative T1-S1 length was $285 \mathrm{~mm}$ and immediate postoperatively was $317 \mathrm{~mm}$. There was 1 late complication, an implant protrusion with an associated infection, in a neuropathic scoliosis patient (Group II) who required implant removal. Conclusion: Our preliminary results suggest that the MCGR system is a safe and effective method. Although the short- and medium-term results are encouraging, further studies are warranted to overcome important and unknown challenges regarding the mechanical behavior of the implant in the long term.

Key words: Early onset scoliosis; magnetically controlled growing rods.

Level of Evidence: IV

Recibido el 14-2-2020. Aceptado luego de la evaluación el 29-5-2020 • Dr. CÉSAR ORELLANA • cesarorellanau@ gmail.com ID https://orcid.org/0000-0001-6755-6298

Cómo citar este artículo: Orellana C, Noel M, Bersusky E, Remondino R, Galaretto E, Tello C. Piantoni L, Francheri Wilson IA. Crecimiento guiado con barras magnéticas en pacientes con escoliosis de inicio temprano. Reporte preliminar. Rev Asoc Argent Ortop Traumatol 2020;85(4):377-386. https://doi.org/10.15417/issn. 1852-7434.2020.85.4.1060 


\section{INTRODUCCIÓN}

Se ha definido a la escoliosis de inicio temprano (EIT) como la deformidad espinal que comienza antes de los 10 años de edad. ${ }^{1,2}$ La EIT incluye un gran número de enfermedades neuromusculares, sindrómicas, idiopáticas y congénitas. ${ }^{3} \mathrm{Si}$ no se la trata, su evolución natural es un aumento progresivo de la deformidad, un eventual compromiso orgánico asociado a un desarrollo anormal de los pulmones, enfermedad restrictiva pulmonar, hipertensión arterial pulmonar y cor pulmonale. ${ }^{4}$

Hay una vasta evidencia publicada sobre la pérdida de crecimiento potencial ocasionada por fusiones tempranas de la columna, especialmente cuando involucran la columna torácica y su repercusión sobre el desarrollo y la función de los pulmones. El crecimiento torácico normal se produce a una velocidad promedio de 1,4 cm por año desde el nacimiento hasta los 5 años de vida; de $0,6 \mathrm{~cm}$ por año, entre los 5 y 10 años de edad, y de 1,2 cm anual, en el adolescente hasta la madurez esquelética.

Por estas razones, se han desarrollado técnicas quirúrgicas que facilitan el crecimiento del tronco y permiten corregir la deformidad y, al mismo tiempo, favorecen el desarrollo del parénquima pulmonar y ponen en un mejor escenario a la columna vertebral y el tórax para su crecimiento. A estos procedimientos se los denomina "de crecimiento guiado" o "de crecimiento amigable" y se llevan a cabo con diferentes sistemas de instrumentación. En esta serie en particular, nos referiremos al uso de barras magnéticas (BM). ${ }^{6}$

La primera serie de casos de EIT y BM data de 2012 y fue publicada por Cheung y cols. ${ }^{7}$ En 2014, la Food and Drug Administration (FDA) de los Estados Unidos autorizó el uso del sistema de barras de crecimiento magnético guiado externamente (Magnetically Controlled Growing Rods; NuVasive CA, EE.UU.). ${ }^{8}$ Estos sistemas tienen ventajas por sobre los sistemas tradicionales de barras de crecimiento. Luego de la instrumentación quirúrgica bajo anestesia general, las distracciones subsiguientes se realizan, en forma ambulatoria, con el paciente habitualmente despierto, en un consultorio externo. ${ }^{9}$ En su gran mayoría, no requieren hospitalización ni anestesia, por lo que disminuyen los riesgos anestésicos, quirúrgicos y asociados a la internación y, a su vez, se evitan la pérdida de días escolares, los problemas sociales y los trastornos psicológicos para el niño y los problemas laborales para su familia. ${ }^{10}$

El objetivo de este estudio fue evaluar nuestra primera serie de pacientes con EIT tratados con el sistema de $\mathrm{BM}$, sus indicaciones, la técnica quirúrgica, los resultados posquirúrgicos y las complicaciones intraoperatorias y posoperatorias.

\section{MATERIALES Y MÉTODOS}

Se analizó, en forma retrospectiva, a una serie de pacientes pediátricos con diagnóstico de EIT, operados con BM en un único centro pediátrico de nivel III, por cinco cirujanos experimentados, durante cuatro años (2014-2018). El análisis estuvo a cargo de un cirujano espinal en formación.

Los criterios de inclusión fueron: pacientes con diagnóstico de EIT, tratados con BM, y con historia clínica e imágenes preoperatorias y posoperatorias completas. El criterio de exclusión fue EIT tratada con otros métodos.

Las variables estudiadas fueron: edad, sexo, etiología, tratamientos previos, procedimientos primarios o conversión a sistema de BM, construcción con barra única o doble, valores angulares (VA) preoperatorios y posoperatorios, distancias radiográficas T1-T12 y T1-S1 preoperatoria y posoperatoria, complicaciones intraoperatorias y posoperatorias.

En los espinogramas postero-anterior y lateral, se analizaron los cambios mediante los VA de Cobb de la curva escoliótica principal, y los VA de cifosis y lordosis antes de colocar las BM y después $(n=19)$. No se evaluaron las curvas compensatorias. Además, se documentaron las variaciones en las distancias T1-T12 y T1-S1 en escala. Las distancias de T1 a T12 y de T1 a S1 se definieron como las distancias comprendidas entre la línea paralela a la placa terminal superior de T1 e inferior a T12, y superior a T1 y superior a S1, respectivamente, en una radiografía de columna completa postero-anterior. ${ }^{11}$ Se documentaron los tipos de sistemas de construcción y los niveles de fijación.

\section{RESULTADOS}

Se evaluó a 19 pacientes (10 niñas [52\%] y 9 niños [48\%]). Todos fueron tratados con el sistema de BM y el seguimiento promedio fue de 2 años y 7 meses (rango de 2 meses a 4 años y 6 meses).

Las etiologías de la EIT eran escoliosis neuromuscular (8 pacientes: 5 con encefalopatía crónica no evolutiva, 2 con atrofia muscular espinal y 1 con miopatía no caracterizada en estudio), escoliosis sindrómica ( 9 pacientes: síndromes de Silver-Russell, William, Marfan, Prader-Willi; 2 casos genéticos en estudio, 2 con displasia esquelética, 1 con neurofibromatosis tipo 1 [Figura 1]), un paciente con escoliosis congénita y uno con escoliosis idiopática infantil (Figura 2). La edad promedio en el momento de la cirugía fue de 7 años y 4 meses (rango 4-12 años). 

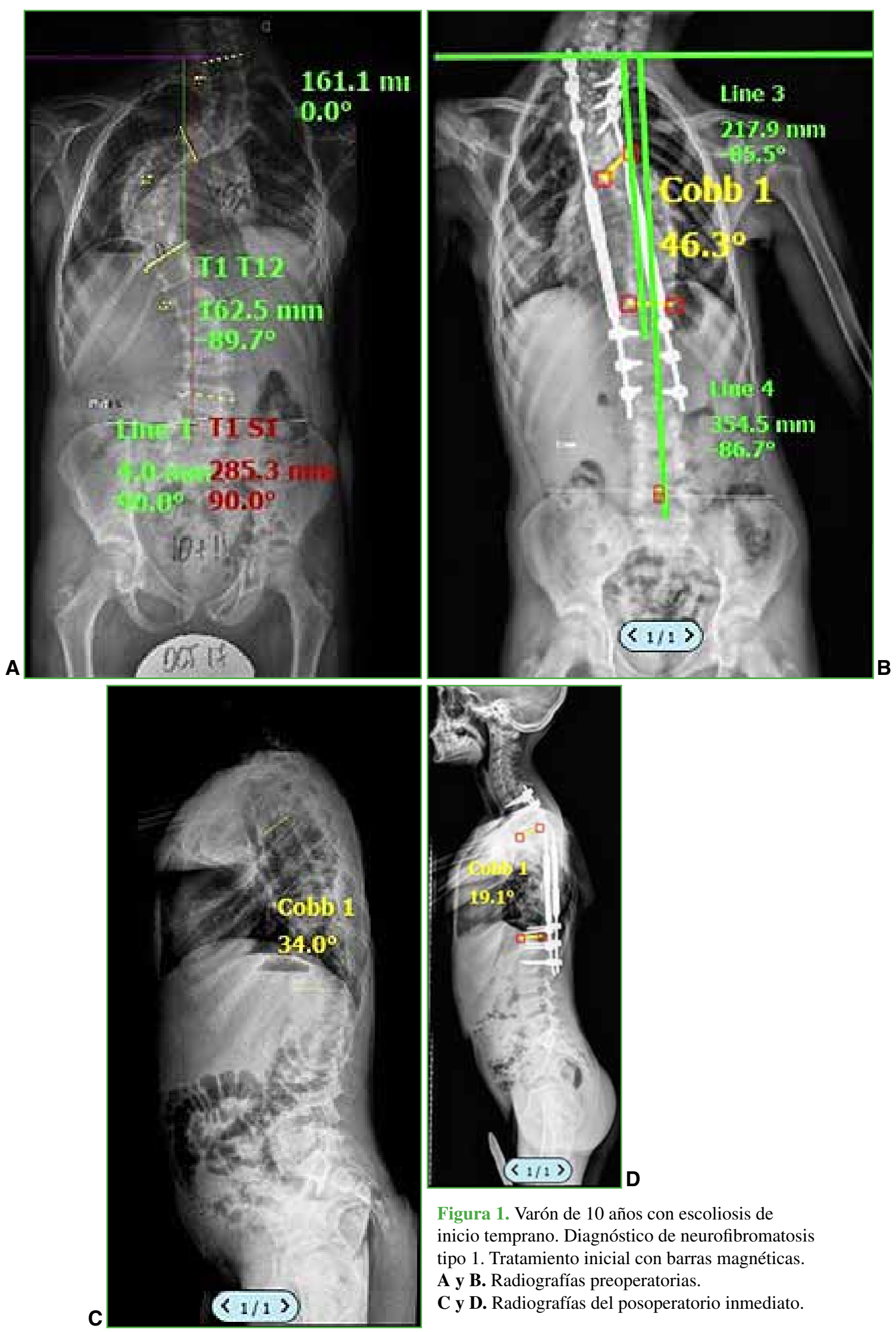

Figura 1. Varón de 10 años con escoliosis de inicio temprano. Diagnóstico de neurofibromatosis tipo 1. Tratamiento inicial con barras magnéticas. A y B. Radiografías preoperatorias.

C y D. Radiografías del posoperatorio inmediato. 


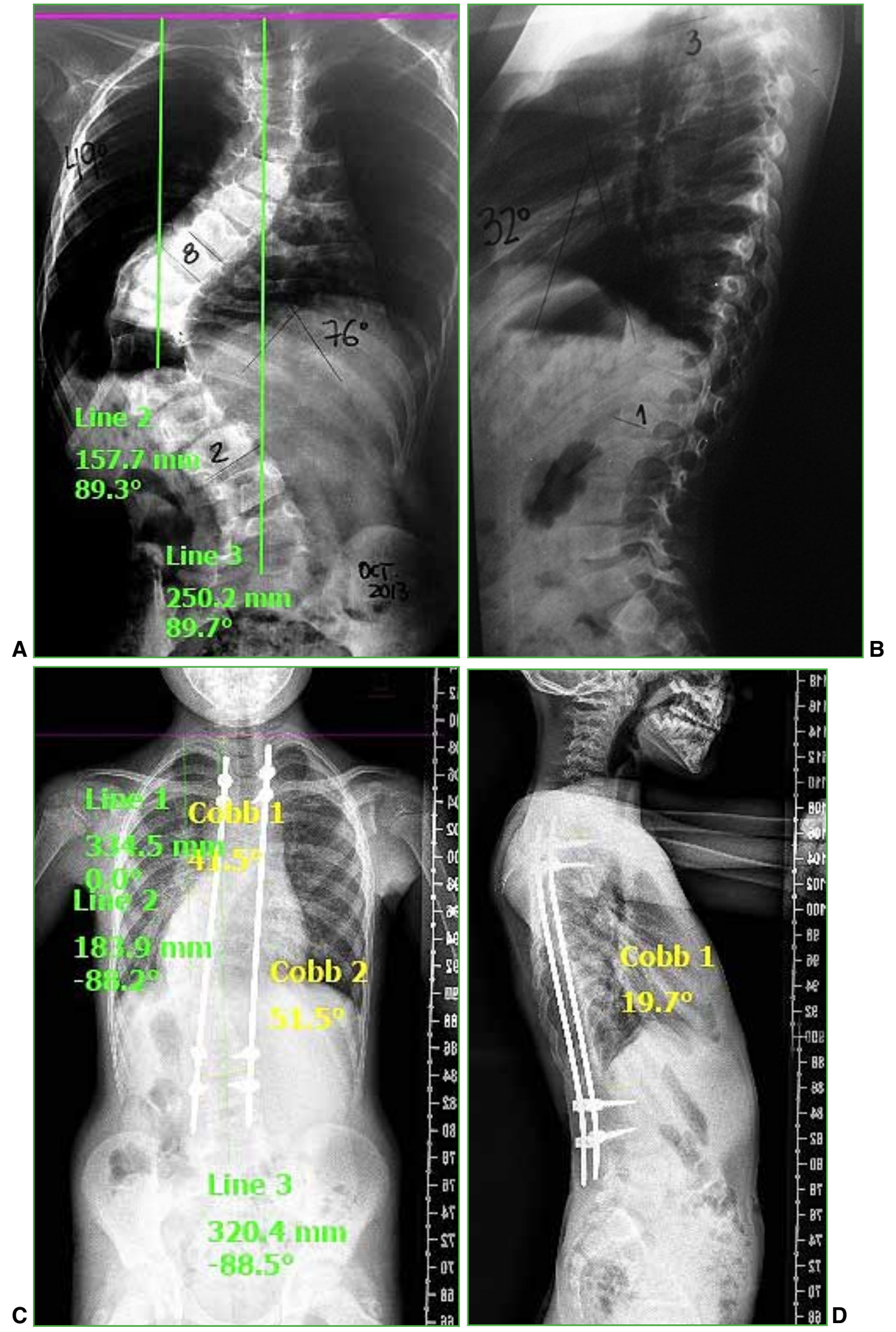

Figura 2. Paciente con diagnóstico de escoliosis idiopática infantil operado a los 5 años de edad, con sistema tradicional a doble barras y conversión a barras magnéticas a los 7 años de edad. A y B. Radiografías preoperatorias. C y D. Radiografías posoperatorias. Tratamiento con sistema tradicional. 

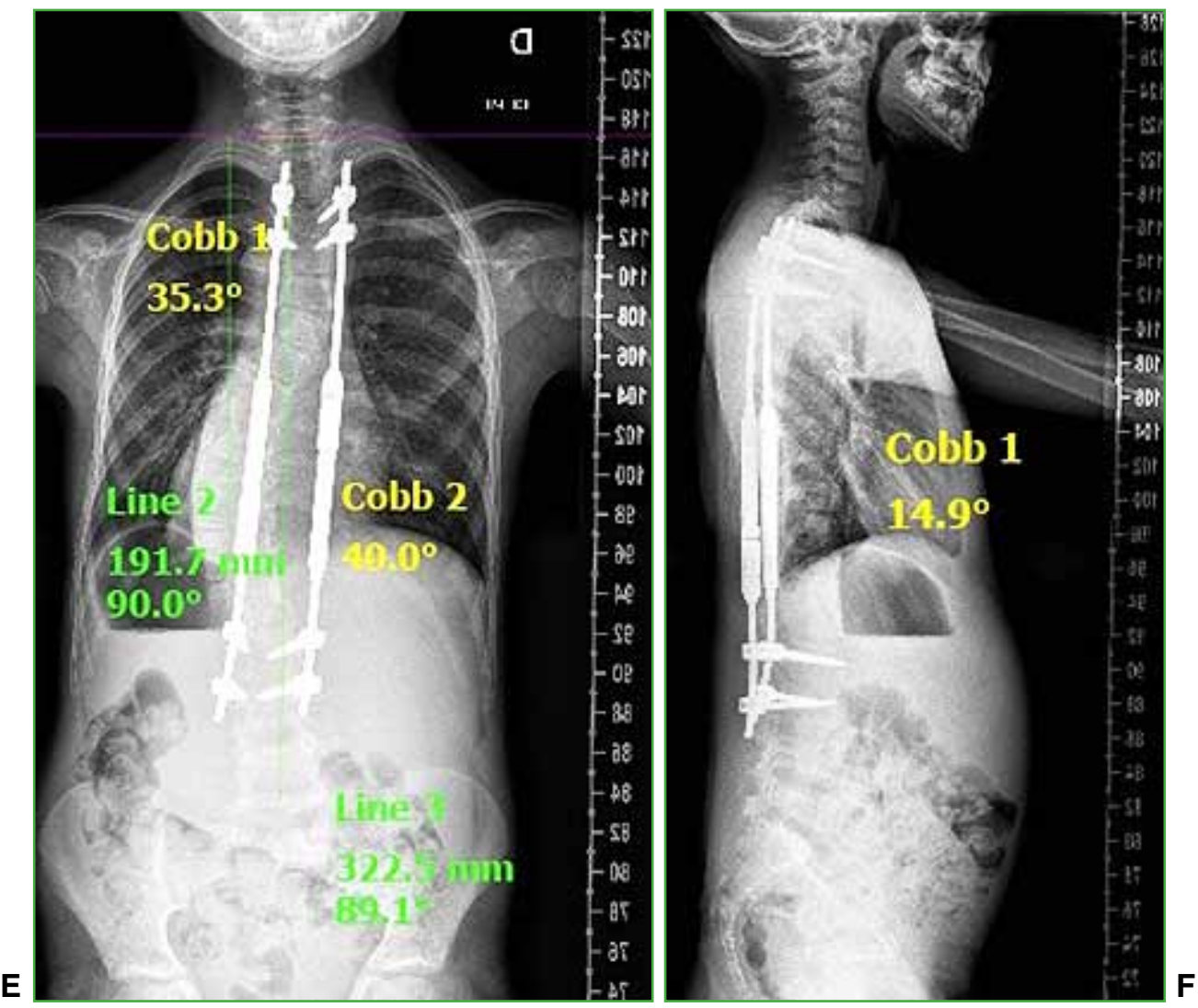

Figura 2. Paciente con diagnóstico de escoliosis idiopática infantil operado a los 5 años de edad, con sistema tradicional a doble barras y conversión a barras magnéticas a los 7 años de edad.

E y F. Radiografías posoperatorias. Conversión a sistema de barras magnéticas.

A 12 pacientes sin tratamientos previos se los operó con el sistema de BM (grupo I), la edad promedio al operarse era de 7 años (rango 4-10). El VA promedio de escoliosis era de $69^{\circ}$ (rango 45-100) antes de la cirugía y de $38^{\circ}$ (rango 17-80) en el posoperatorio inmediato; y el de cifosis era de $58^{\circ}$ (rango 22-111) y de $36^{\circ}$ (rango 7-82), respectivamente.

La distancia promedio T1-T12 preoperatoria era de $147 \mathrm{~mm}$ (rango 95-190) y de $169 \mathrm{~mm}$ (rango 104-217) en el posoperatorio inmediato. La distancia promedio T1-S1 preoperatoria era de $253 \mathrm{~mm}$ (rango 205-288) y de 306 $\mathrm{mm}$ (rango 215-354) en el posoperatorio inmediato (Tabla 1).

Se realizó una conversión de un sistema de distracción tradicional a un sistema de BM (grupo II) en siete pacientes. Dos conversiones a BM se efectuaron por complicaciones (un aflojamiento de tornillos proximales y una ruptura de barra) y cuatro, en pacientes con escoliosis sindrómicas. El caso restante era un paciente con escoliosis idiopática infantil tratado con corsé de yeso bajo anestesia, sucesivos corsés termplásticos y barras de crecimiento tradicionales desde los 2 años de vida, al agotar la posibilidad de elongación, se decidió la conversión a BM (Figura 2).

En el grupo II, la edad promedio al operarse fue de 7 años (rango 4-12). El VA promedio de escoliosis era de $56^{\circ}$ (rango 39-101) antes de la cirugía y de $46^{\circ}$ (rango 30-76) en el posoperatorio inmediato; y el de cifosis era de $39^{\circ}$ (rango $7-81$ ) y $32^{\circ}$ (rango 4-52), respectivamente. La distancia promedio T1-T12 preoperatoria era de $174 \mathrm{~mm}$ (rango 117-275) y de $183 \mathrm{~mm}$ (rango 138-275) en el posoperatorio inmediato. La distancia promedio T1-S1 preoperatoria era de $317 \mathrm{~mm}$ (rango 234-507) y de $329 \mathrm{~mm}$ (rango 249-507) en el posoperatorio inmediato (Tabla 2). 
Tabla 1. Grupo I: Escoliosis de inicio temprano sin tratamiento previo

\begin{tabular}{|c|c|c|c|c|c|c|c|c|c|c|c|}
\hline \multirow{2}{*}{$\begin{array}{l}\text { Pa- } \\
\text { ciente }\end{array}$} & \multirow[t]{2}{*}{ Etiología } & \multirow[t]{2}{*}{ Edad } & \multicolumn{4}{|c|}{ Preoperatorio } & \multicolumn{5}{|c|}{ Posoperatorio } \\
\hline & & & COBB & Cifosis & $\begin{array}{l}\text { T1- } \\
\text { T12 }\end{array}$ & $\begin{array}{l}\text { T1- } \\
\text { S1 }\end{array}$ & COBB & Cifosis & $\begin{array}{l}\text { T1- } \\
\text { T12 }\end{array}$ & $\begin{array}{l}\text { T1- } \\
\text { S1 }\end{array}$ & $\begin{array}{l}\text { Complica- } \\
\text { ciones }\end{array}$ \\
\hline 1 & Síndrome de Williams & 6 & 70 & 37 & 143 & 258 & 36 & 11 & 170 & 335 & - \\
\hline 2 & Neurofibromatosis tipo 1 & 10 & 98 & 28 & 162 & 258 & 46 & 19 & 217 & 354 & - \\
\hline 3 & ECNE & 7 & 47 & 60 & 159 & 247 & 43 & 60 & 183 & 297 & - \\
\hline 4 & ECNE & 7 & 100 & 111 & 157 & 242 & 80 & 77 & 171 & 293 & - \\
\hline 5 & ECNE & 4 & 95 & 86 & 95 & 205 & 75 & 82 & 104 & 215 & - \\
\hline 6 & Escoliosis neuropática & 5 & 91 & 51 & 118 & 210 & 29 & 17 & 154 & 271 & - \\
\hline 7 & Síndrome de displasia ósea & 9 & 46 & 22 & 190 & 313 & 17 & 42 & 168 & 313 & - \\
\hline 8 & Síndrome de Prader-Willi & 6 & 65 & 64 & 160 & 278 & 22 & 31 & 171 & 312 & - \\
\hline 9 & Escoliosis miopática & 9 & 68 & 22 & 162 & 288 & 22 & 11 & 202 & 351 & - \\
\hline 10 & $\begin{array}{l}\text { Síndrome de atrofia } \\
\text { muscular espinal }\end{array}$ & 7 & 45 & 103 & 132 & 212 & 36 & 40 & 168 & 321 & - \\
\hline 11 & $\begin{array}{l}\text { Síndrome de atrofia } \\
\text { muscular espinal }\end{array}$ & 7 & 60 & 60 & 136 & 256 & 23 & 7 & 170 & 333 & - \\
\hline 12 & Escoliosis congénita & 10 & 45 & 55 & 157 & 271 & 36 & 36 & 157 & 283 & - \\
\hline \multicolumn{2}{|l|}{ Valor $\mathrm{n}$} & 7,2 & 69,2 & 58,2 & 147,6 & 253,2 & 38,7 & 36,1 & 169,6 & 306,5 & 0 \\
\hline
\end{tabular}

$\mathrm{ECNE}=$ encefalopatía crónica no evolutiva.

Tabla 2. Grupo II: Escoliosis de inicio temprano tratada con conversión de sistemas tradicionales a barras magnéticas

\begin{tabular}{|c|c|c|c|c|c|c|c|c|c|c|c|c|}
\hline \multirow[t]{3}{*}{ Paciente } & \multirow[t]{3}{*}{ Etiología } & \multirow[t]{3}{*}{ Edad } & \multicolumn{4}{|c|}{ Preoperatorio } & \multicolumn{6}{|c|}{ Posoperatorio } \\
\hline & & & \multirow[t]{2}{*}{ COBB } & \multirow[t]{2}{*}{ Cifosis } & \multirow{2}{*}{$\begin{array}{l}\mathrm{T} 1- \\
\mathrm{T} 12\end{array}$} & \multirow{2}{*}{$\begin{array}{l}\text { T1- } \\
\text { S1 }\end{array}$} & \multirow[t]{2}{*}{ COBB } & \multirow{2}{*}{$\begin{array}{c}\text { Cifo- } \\
\text { sis }\end{array}$} & \multirow{2}{*}{$\begin{array}{l}\text { T1- } \\
\text { T12 }\end{array}$} & \multirow{2}{*}{$\begin{array}{l}\text { T1- } \\
\text { S1 }\end{array}$} & \multicolumn{2}{|c|}{ Complicaciones } \\
\hline & & & & & & & & & & & Barras & $\begin{array}{c}\text { Barras } \\
\text { magnéticas }\end{array}$ \\
\hline 1 & $\begin{array}{l}\text { Síndrome de } \\
\text { Silver-Russell }\end{array}$ & 12 & 39 & 33 & 275 & 507 & 39 & 33 & 275 & 507 & & \\
\hline 2 & ECNE & 4 & 101 & 50 & 117 & 234 & 76 & 50 & 138 & 249 & & $\begin{array}{l}\text { Retiro del } \\
\text { implante }\end{array}$ \\
\hline 3 & $\begin{array}{l}\text { Escoliosis } \\
\text { sindrómica }\end{array}$ & 9 & 67 & 56 & 168 & 283 & 59 & 52 & 172 & 292 & $\begin{array}{l}\text { Aflojamiento } \\
\text { de tornillos } \\
\text { proximales }\end{array}$ & \\
\hline 4 & $\begin{array}{l}\text { Síndrome de } \\
\text { Marfan }\end{array}$ & 5 & 44 & 7 & 182 & 309 & 43 & 4 & 173 & 315 & & \\
\hline 5 & $\begin{array}{l}\text { Escoliosis } \\
\text { idiopática } \\
\text { juvenil }\end{array}$ & 7 & 43 & 11 & 185 & 334 & 40 & 14 & 191 & 341 & & \\
\hline 6 & $\begin{array}{l}\text { Condrodis- } \\
\text { plasia }\end{array}$ & 9 & 51 & 81 & 122 & 238 & 30 & 46 & 158 & 280 & $\begin{array}{l}\text { Ruptura de } \\
\text { barra }\end{array}$ & \\
\hline 7 & $\begin{array}{l}\text { Síndrome de } \\
\text { Escobar }\end{array}$ & 8 & 48 & 41 & 170 & 317 & 38 & 30 & 180 & 319 & & \\
\hline Valor med & & 7,7 & 56,1 & 39,8 & 174,1 & 317,4 & 46,4 & 32,7 & 183,8 & 329 & 2 & 1 \\
\hline
\end{tabular}

$\mathrm{ECNE}=$ encefalopatía crónica no evolutiva. 
Las construcciones fueron con doble barra ( 13 casos) y con barra única (6 casos). El promedio de niveles instrumentados fue de 5 (rango 4-6).

En esta serie de casos, se produjo una complicación alejada: exposición del implante e infección profunda por Staphylococcus aureus, en un paciente con escoliosis neuropática del grupo II, que requirió limpieza, desbridamiento y retiro del implante.

\section{DISCUSIÓN}

El sistema de distracción con BM fue diseñado como una opción más de tratamiento para las deformidades de la columna de inicio temprano, una de sus ventajas es que se pueden realizar repetidas elongaciones no invasivas, sin necesidad de procedimientos anestésicos en la gran mayoría de los casos. A su vez, conserva las funciones de las técnicas tradicionales de elongación, como preservar el crecimiento de la columna vertebral, el tórax y los pulmones. ${ }^{9}{ }^{12}$ Su colocación es técnicamente similar a la de un procedimiento convencional, y sus ventajas se observan en el posoperatorio, permite hacer distracciones no invasivas, ambulatorias y con el paciente despierto; rara vez, es necesario administrar una sedación menor a niños muy pequeños. El primer paso consiste en localizar el elemento de distracción telescópico (actuator) con un imán; a continuación, se coloca el imán externamente, antes se lo calibra a los milímetros por distraer sobre la zona del actuator de la(s) barra(s); el procedimiento dura unos pocos segundos; a veces, se puede escuchar el clic del implante ante la maniobra de distracción. ${ }^{9}$ El procedimiento no requiere hospitalización ni anestesia y así se evitan los riesgos anestésicos, quirúrgicos y propios de la internación; también disminuyen los días de clase perdidos y los trastornos psicosociales para los niños y los problemas laborales para las familias..$^{10}$ En nuestra serie, solo dos pacientes tuvieron dolor durante la distracción magnética que obligó a dar marcha atrás, parcialmente, al procedimiento de elongación, con distracciones de $3 \mathrm{~mm}$ en cada una de ellas; los síntomas desaparecieron sin necesidad de internación o analgesia. Luego ambos pacientes continuaron con los planes de distracción sin sintomatología.

Se han comunicado complicaciones en el sistema de BM a los dos años de seguimiento: ruptura de barras, ruptura del actuator, aflojamiento de anclajes, metalosis local y aumento de titanio y vanadio en sangre, infección de la herida quirúrgica, entre otras. ${ }^{10,13,14}$ En esta serie, fue necesario retirar el implante por protrusión e infección profunda de la herida en un único paciente con escoliosis neuropática del grupo II; en consecuencia, la tasa de complicaciones fue del $5 \%$.

Los pacientes tratados con sistemas de crecimiento tradicionales necesitan distracciones quirúrgicas, como promedio, cada 6-12 meses. Estos procedimientos requieren de repetidas anestesias e intervenciones quirúrgicas, lo que incrementa el riesgo de complicaciones, como infección superficial o profunda, fusión espontánea de los segmentos instrumentados, ruptura de las barras, aflojamiento o prominencia del implante, trastornos psicológicos (estrés postraumático, depresión por repetidos procedimientos). Además, se deben considerar los costos indirectos para los niños y los padres como resultado del ausentismo escolar y laboral, respectivamente. ${ }^{6,10,12,15,16}$

Bess y cols. publicaron una serie de 140 casos tratados mediante el sistema de barras de crecimiento convencionales con una tasa de complicaciones del $58 \% .{ }^{13}$ En una serie de 28 pacientes con BM, Beaven y cols. comunicaron una tasa de complicaciones del $29 \% .{ }^{9}$ En una revisión bibliográfica sistemática, se halló una tasa global de complicaciones del $46 \%$ en pacientes tratados con BM y un seguimiento mínimo de dos años. ${ }^{17}$

En un estudio comparativo entre el sistema de BM (10 pacientes) y barras tradicionales (10 pacientes), se observó una importante reducción en el número de cirugías con el sistema de BM. ${ }^{18}$

Cualquier cirugía de revisión implica un aumento de la morbilidad para el niño, como así también un perjuicio financiero significativo tanto para la familia como para el sistema de salud. El sistema de BM tiene un costo inicial excesivo, pero genera un ahorro a partir del tercer año de la implantación, si se lo compara con las barras de crecimiento convencionales. ${ }^{19,20}$

No hay consenso en cuanto a los tiempos y la cantidad de milímetros por distraer en cada procedimiento. Nuestro protocolo consistió en distracciones cada tres meses, con una elongación promedio de 3 a 5 mm en cada sesión; ${ }^{17,21}$ esto implicaría a cuatro distracciones anuales y un promedio de elongación anual del sistema de 12-20 mm.

Un estudio de Rushton y cols. ha determinado qué proporción de las BM implantadas fueron capaces de reproducir la misma fuerza de distracción con respecto a las barras no implantadas, declaradas por el fabricante. De 45 BM utilizadas durante una media de 2 años y 7 meses, 10 (22\%) produjeron una fuerza superior o igual a la referida por el fabricante; seis (13\%), una fuerza menor y 29 (64\%) no produjeron ninguna fuerza de distracción. ${ }^{22}$ 
Otro factor importante por tener en cuenta son las construcciones de barra única y de barra doble. El sistema de doble barra lograría una mayor estabilidad y un mejor control mecánico de la columna vertebral; ${ }^{23,24}$ por otro lado, muchas veces, el tamaño del paciente y la cobertura cutánea pueden no brindar óptimas condiciones para el uso de doble barra y se opta por un sistema de barra única. En nuestro estudio, 13 casos fueron construcciones a doble barra y seis, a barra única.

Luego del procedimiento de distracción en el consultorio externo, se toman radiografías de control para constatar los milímetros de distracción, el control de anclajes y la corrección de la curva. En los últimos tiempos, se ha descrito un nuevo sistema de medición bajo control ecográfico para controlar el alargamiento y reducir el riesgo de una excesiva radiación. ${ }^{7,25}$ Hemos comenzado a usar este nuevo método en nuestra práctica. Otorga una idea estimada de distracción en milímetros; algunas de sus limitaciones son: no valorar el estado del implante ni el VA de la curva y estar sujeto a la variabilidad interobservador (estudio dependiente del técnico); por este motivo, creemos que es necesario tomar una radiografía, al menos, una vez al año.

Entre las limitaciones de este estudio, podemos mencionar: el escaso número de casos, la heterogeneidad de la muestra y el corto seguimiento posoperatorio.

\section{CONCLUSIONES}

En esta serie de casos, los resultados preliminares del sistema de distracción con BM en el crecimiento guiado de la EIT indican que mantiene los beneficios del crecimiento torácico y espinal, es un método no invasivo y ambulatorio, que no requiere anestesia y con una tasa de complicaciones menor. Es una alternativa al sistema de barras de crecimiento tradicionales en pacientes con alta morbilidad y un riesgo quirúrgico alto.

Nuestros resultados preliminares sugieren que es un método seguro y eficaz. Si bien los resultados a corto y mediano plazo son alentadores, todavía quedan desafíos importantes e incógnitas respecto del comportamiento mecánico del implante en un largo período de seguimiento.

Conflicto de intereses: Los autores no declaran conflictos de intereses.

ORCID de M. Noel: https://orcid.org/0000-0002-6668-1342 ORCID de E. Bersusky: https://orcid.org/0000-0002-3121-9326 ORCID de R. Remondino: https://orcid.org/0000-0002-8388-298X ORCID de E. Galaretto: https://orcid.org/0000-0003-4121-8228
ORCID de C. Tello: https://orcid.org/0000-0002-1733-1004

ORCID de L. Piantoni: https://orcid.org/0000-0002-6616-9314

ORCID de I. A. Francheri Wilson: https://orcid.org/0000-0003-2634-0719

\section{BIBLIOGRAFÍA}

1. Skaggs DL, Guillaume T, El-Hawary R, Emans J, Mendelow M, Smith J. Early Onset Scoliosis Consensus Statement, SRS Growing Spine Committee. Spine Deform 2015;3(2):107. https://doi.org/10.1016/j.jspd.2015.01.002

2. Williams BA, Matsumoto H, McCalla JD, Akbarnia BA, Blakemore LC, Betz RR, et al. Development and initial validation of the classification of early onset scoliosis (C-EOS). J Bone Joint Surg Am 2014;96(16):1359-67. https://doi.org/10.2106/JBJS.M.00253

3. Campbell RM Jr, Smith MD. Thoracic insufficiency syndrome and exotic scoliosis. J Bone Joint Surg Am 2007;89(Suppl):108-22. https://doi.org/10.2106/JBJS.F.00270

4. Pehrsson K, Larsson S, Nachemson A. Long-term follow-up of patients with untreated scoliosis. A study of mortality, causes of death, and symptoms. Spine 1992;17(9):1091-6. https://doi.org/10.1097/00007632-199209000-00014

5. Vitale MG, Matsumoto H, Roye DP Jr., Bye MR, Gomez JA, Booker WA, et al. Pulmonary function, radiographic measures, and quality of life in children with congenital scoliosis: an evaluation of patient outcomes after early spinal fusion. Spine (Phila Pa 1976) 2008;33(11):1242-9. https://doi.org/10.1097/BRS.0b013e3181714536 
6. Sankar WN, Acevedo DC, Skaggs DL. Comparison of complications among growing spinal implants. Spine (Phila Pa 1976) 2010;35(23):2091-6. https://doi.org/10.1097/BRS.0b013e3181c6edd7

7. Cheung KM, Cheung JP, Samartzis D, Mak K, Wong Y, Akbarnia BA, et al. Magnetically controlled growing rods for severe spinal curvature in young children: a prospective case series. Lancet 2012;379:1967-74. https://doi.org/10.1016/S0140-6736(12)60112-3

8. Skov ST, Wijdicks SPJ, Bünger C, Castelein RM, Li H, Kruyt MC. Treatment of early-onset scoliosis with a hybrid of a concave magnetic driver (magnetic controlled growth rod) and a contralateral passive sliding rod construct with apical control: preliminary report on 17 cases. Spine J 2018;18(1):122-9. https://doi.org/10.1016/j.spinee.2017.06.027

9. Beaven A, Gardner AC, Spilsbury JB, Marks DS, Mehta JS, Newton-Ede M. Magnetically controlled growing rods: the experience of mechanical failure from a single center consecutive series of 28 children with a minimum followup of 2 years. Asian Spine J 2018;12(5):794-802. https://doi.org/10.31616/asj.2018.12.5.794

10. Dannawi Z, Altaf F, Noordeen H, Harshavardhana NS, El Sebaie H. Early results of a remotely-operated magnetic growth rod in early-onset scoliosis. Bone Joint J Br 2013;95(1):75-80. https://doi.org/10.1302/0301-620X.95B1.29565

11. Akbarnia BA, Pawelek JB, Sponseller PD, Cheung KM, Demirkiran G, Hazem El Sebaie H. et al. Traditional growing rods versus magnetically controlled growing rods for the surgical treatment of early-onset scoliosis: a casematched 2-year study. Spine Deform 2014;2(6):493-7. https://doi.org/10.1016/j.jspd.2014.09.050

12. Bekmez S, Dede O, Yazici M. Advances in growing rods treatment for early onset scoliosis. Curr Opin Pediatr 2017;29(1):87-93. https://doi.org/10.1097/MOP.0000000000000432

13. Bess S, Akbarnia BA, Skaggs DL, Thompson GH, Sponseller PD, Shah SA, et al. Complications of growingrod treatment for early-onset scoliosis: analysis of one hundred and forty patients. J Bone Joint Surg Am 2010;92(15):2533-43. https://doi.org/10.2106/JBJS.I.01471

14. La Rosa G, Oggiano L, Ruzzini L. Magnetically controlled growing rods for the management of early-onset scoliosis: a preliminary report. J Pediatr Orthop 2017;37(2):79-85. https://doi.org/10.1097/BPO.0000000000000597

15. Yilmaz G, Huri G, Demirkran G, Dalolu K, Ozkan C, Alanay A, et al. The effect of posterior distraction on vertebral growth in immature pigs: an experimental simulation of growing rod technique. Spine (Phila Pa 1976) 2010;35(7):730-3. https://doi.org/10.1097/BRS.0b013e3181bcc3a3

16. Tello CA. Harrington instrumentation without arthrodesis and consecutive distraction program for young children with severe spinal deformities. Experience and technical details. Orthop Clin North Am 1994;25:333-51. PMID: 8159406

17. Wu AM, Cheung JPY, Cheung KMC, Lin JL, Jin HM, Chen D, et al. Minimum 2-year experience with magnetically controlled growing rods for the treatment of early-onset scoliosis: a systematic review. Asian Spine J 2019; 13(4): 682-93. https://doi.org/10.31616/asj.2018.0272

18. Bekmez S, Afandiyev A, Dede O, Karaismailolu E, Demirkiran HG, Yazici M. Is magnetically controlled growing rod the game changer in early-onset scoliosis? A preliminary report. J Pediatr Orthop 2019;39(3):e195-e200. https://doi.org/10.1097/bpo.0000000000001268

19. Rolton D, Richards J, Nnadi C. Magnetic controlled growth rods versus conventional growing rod systems in the treatment of early onset scoliosis: a cost comparison. Eur Spine J 2015;24:1457-61. https://doi.org/10.1007/s00586-014-3699-7

20. Jenks M, Craig J, Higgins J, Willits I, Barata T, Wood H, et al. The MAGEC system for spinal lengthening in children with scoliosis: a NICE Medical Technology Guidance. Appl Health Econ Health Policy 2014;12:587-99. https://doi.org/10.1007/s40258-014-0127-4

21. Cheung JPY, Yiu K, et al. Mean 6-year follow-up of magnetically controlled growing rod patients with early onset scoliosis: a glimpse of what happens to graduates. Neurosurgery 2019;84(5):1112-23. https://doi.org/10.1093/neuros/nyy270

22. Rushton PRP, Smith SL, Forbes L, Bowey AJ, Gibson MJ, Joyce TJ. Force testing of explanted magnetically controlled growing rods. Spine (Phila Pa 1976) 2019;44(4):233-39. https://doi.org/10.1097/BRS.0000000000002806

23. Thompson GH, Akbarnia BA, Campbell RM Jr. Growing rod techniques in early-onset scoliosis. J Pediatr Orthop 2007;27:354-61. https://doi.org/10.1097/BPO.0b013e3180333eea 
24. Heydar AM, Sirazi S, Bezer M. Magnetic controlled growing rods as a treatment of early onset scoliosis: early results with two patients. Spine (Phila Pa 1976) 2016;41(22):E1336-E1342. https://doi.org/10.1097/BRS.0000000000001614

25. Stokes OM, O’Donovan EJ, Samartzis D, Bow CH, Luk KDK, Cheung KMC. Reducing radiation exposure in earlyonset scoliosis surgery patients: novel use of ultrasonography to measure lengthening in magnetically-controlled growing rods. Spine J 2014;14:2397-2404. https://doi.org/10.1016/j.spinee.2014.01.039 